\title{
Saint-John Perse, Correspondance avec Henri Hoppenot (1915-1975)
}

\section{Francesca Pagani}

\section{Q OpenEdition}

1 Journals

\section{Edizione digitale}

URL: http://journals.openedition.org/studifrancesi/6424

DOI: ERREUR PDO dans/localdata/www-bin/Core/Core/Db/Db.class.php L.34 : SQLSTATE[HYO00]

[2006] MySQL server has gone away

ISSN: 2421-5856

\section{Editore}

Rosenberg \& Sellier

\section{Edizione cartacea}

Data di pubblicazione: 1 novembre 2010

Paginazione: 587

ISSN: 0039-2944

\section{Notizia bibliografica digitale}

Francesca Pagani, «Saint-John Perse, Correspondance avec Henri Hoppenot (1915-1975)», Studi Francesi [Online], 162 (LIV | III) | 2010, online dal 30 novembre 2015, consultato il 07 janvier 2021. URL: http:// journals.openedition.org/studifrancesi/6424 ; DOI: https://doi.org/10.4000/studifrancesi.6424

Questo documento è stato generato automaticamente il 7 janvier 2021.

\section{(c)}

Studi Francesi è distribuita con Licenza Creative Commons Attribuzione - Non commerciale - Non opere derivate 4.0 Internazionale. 


\title{
Saint-John Perse, Correspondance avec Henri Hoppenot (1915-1975)
}

\author{
Francesca Pagani
}

\section{NOTIZIA}

SAINT JOHN PERSE, Correspondance avec Henri Hoppenot (1915-1975), édition établie, annotée et présentée par Marie France Mousli, Paris, Gallimard, 2009 ( «Cahiers Saint-John Perse», 19), pp. 247.

1 Questo volume della serie «Saint-John Perse»-ultimo frutto dell'opera più che trentennale intrapresa da parte dell'omonima fondazione in seno alla collana dei «Cahiers de la NRF» -, propone un'edizione estremamente curata del carteggio tra Alexis Léger (conformiamo l'ortografia a quella adottata nell'opera) e Henri Hoppenot. Il futuro Saint-John Perse conobbe Hoppenot nell'estate del 1914 presso l'Ufficio stampa del Ministero degli Esteri: per entrambi si trattava dell'esordio di una carriera diplomatica destinata a riservare loro antitetiche Fortune e di un'amicizia coltivata per più di sessant'anni. Le vicissitudini personali e i frequenti trasferimenti in paesi lontani (dalla Cina agli Stati Uniti) non seppero infatti scalfire un'intesa che, malgrado la dichiarata disposizione di Léger al silenzio, ben si evince da questa corrispondenza sinora inedita - le opere di Saint-John Perse pubblicate nella collana della Biblio-thèque de la Pléiade, infatti, non la contemplano.

2 Marie France Mousli, curatrice di questo volume e del carteggio tra Hélène e Henri Hoppenot e Darius e Madeleine Milhaud (Gallimard, 2005), delinea nella sua introduzione il percorso biografico degli autori e individua l'intreccio della loro corrispondenza, ricostruita attraverso i fondi presenti nella biblioteca Jacques Doucet, dove gli stessi coniugi Hoppenot depositarono il carteggio di Léger nel 1973, e quelli in possesso della Fondazione Saint-John Perse presso la biblioteca Méjanes della Cité du livre di Aix-en-Provence. 
3 La curatrice propone il carteggio, che si compone di settantotto lettere, cartoline e telegrammi, suddividendolo cronologicamente in sette sezioni, ognuna delle quali si apre con una presentazione che ha il pregio di contestualizzare il corpus epistolare evitando il supporto di un apparato di note troppo denso e rendendo così la lettura più lineare e assai gradevole. Un'ulteriore sezione è dedicata al carteggio tra i coniugi Hoppenot e la famiglia Léger, nelle persone della madre e della moglie del poeta, e tra Hoppenot e coloro che gravitarono intorno alla candidatura al Nobel di Saint-John Perse, che il diplomatico amico di Léger supportò intensamente.

4 Emerge dal carteggio una dimensione intima che, spaziando dalle questioni private a quelle politiche, permette di mettere in luce aspetti poco conosciuti della vita di SaintJohn Perse, attraverso la profondità di un legame che egli non mancò di attestare: «Il y a de l'élégance de la part du Sort à faire aussi bien les choses en réservant mystérieusement ce rôle à une amitié comme la vôtre. Elle ne date pas d'hier, et la vie, qui l'a nouée, l'a très humainement nourrie, à travers bien des événements. Je demeure sensible au sens que prend pour moi, dans l'allongement des ombres sous nos pas, la portée de ce lien vivant» (p. 126).

5 Chiude il volume un aggiornamento della bibliografia dedicata a Saint-John Perse nel triennio 2005-2008, così come un preciso indice dei nomi e delle persone. Da segnalare un pregevole apparato iconografico che riproduce alcune lettere presenti nel carteggio e alcune fotografie appartenenti alla Fondazione Saint-John Perse e ad archivi privati. 